LAPTH-1185/07

\title{
Black hole mass and angular momentum in topologically massive gravity
}

\author{
Adel Bouchareb* and Gérard Clément ${ }^{\dagger}$ \\ Laboratoire de Physique Théorique LAPTH (CNRS), \\ B.P.110, F-74941 Annecy-le-Vieux cedex, France
}

31 May 2007

\begin{abstract}
We extend the Abbott-Deser-Tekin approach to the computation of the Killing charge for a solution of topologically massive gravity (TMG) linearized around an arbitrary background. This is then applied to evaluate the mass and angular momentum of black hole solutions of TMG with non-constant curvature asymptotics. The resulting values, together with the appropriate black hole entropy, fit nicely into the first law of black hole thermodynamics.
\end{abstract}

\footnotetext{
*Email: bouchare@lapp.in2p3.fr

${ }^{\dagger}$ Email: gclement@lapp.in2p3.fr
} 


\section{Introduction}

A rich, ghost-free theory of gravity in $2+1$ dimensions is topologically massive gravity [1], also known as Chern-Simons gravity. This is defined by the field equations

$$
G^{\mu}{ }_{\nu}+\frac{1}{\mu} C_{\nu}^{\mu}=\mathrm{E} g_{\mu \nu}
$$

where $G^{\mu}{ }_{\nu} \equiv R^{\mu}{ }_{\nu}-\frac{1}{2} R \delta^{\mu}{ }_{\nu}$ is the Einstein tensor,

$$
C_{\nu}^{\mu} \equiv \frac{1}{\sqrt{|g|}} \epsilon^{\mu \alpha \beta} D_{\alpha}\left(R_{\beta \nu}-\frac{1}{4} g_{\beta \nu} R\right)
$$

is the Cotton tensor $\left(\epsilon^{\mu \alpha \beta}\right.$ is the antisymmetrical symbol), $\mathrm{E}$ is the cosmological constant and $\mu$ is the topological mass constant.

TMG is known to admit two kinds of black hole solutions. The first is the well-known asymptotically AdS BTZ solution [2] of Einstein gravity with a negative cosmological constant, which has constant curvature and thus also solves trivially TMG [3]. The second is the non-asymptotically flat, non-asymptotically AdS, $\mathrm{E}=0$ ACL black hole solution [4] 1 . The computation of the mass and angular momentum of these black holes presents an interesting challenge. The ususal ADM approach [7] fails because these solutions are not asymptotically flat. In the quasilocal energy approach 8, one derives canonically a hamiltonian, given by the sum of a bulk integral, which vanishes on shell, and of a surface term. The quasilocal energy is the difference between the on-shell value of the Hamiltonian and its value for a suitably chosen "vacuum" solution. However this approach cannot be at present used for our purpose, as the canonical formulation of TMG is given in [9, 10] only modulo unknown surface terms [4, 11].

In the super angular momentum (SAM) approach [12], a theory of $2+1$ gravity with two Killing vectors is reduced to a mechanical system with the $S L(2, R)$ invariance, and two specific constants of the motion of this system are identified as the mass and angular momentum of the associated $(2+1)$ dimensional gravitating configuration. This super angular momentum approach was shown in 12 to coincide with the quasilocal approach for generic Einstein-scalar field theories in $2+1$ dimensions. It was applied in [4] to compute the mass and angular momentum of the BTZ and ACL black holes. The SAM values for the BTZ black hole (as a solution of TMG) are consistent with those given by Garcia et al. [13] and others [14, 15, 16, 17, 18].

\footnotetext{
${ }^{1}$ Related solutions with horizons were previously given in [5, 6], but these violate causality, due to closed timelike circles outside the horizon, and so are not regular black holes.
} 
On the other hand, the SAM values for the ACL black hole are not consistent with the first law of black hole thermodynamics, which raises doubts about their validity. Inspection of Eq. (28) of [4] shows that agreement with the first law is restored if the SAM value for the angular momentum is kept unchanged, while the SAM value for the mass is doubled. Thus one expects that the correct mass of the ACL black hole should be twice the 'naive' SAM mass, as will be checked in Sect. 4 of the present paper. We note that this factor of 2 problem with the SAM mass of the ACL black hole is curiously reminiscent of the well-known problem with the Møller-Komar energy in four-dimensional general relativity [19, 20] (see the discussion in [21]): the Komar superpotential gives the correct angular momentum, but only half of the correct energy.

An approach to compute the energy of asymptotically AdS solutions to cosmological gravity was given by Abbott and Deser [22], and extended by Deser and Tekin to the computation of the energy of asymptotically dS or AdS solutions to higher curvature gravity theories [23] and to topologically massive gravity [24]. In this Abbott-Deser-Tekin (ADT) approach, the field equations are linearized around an appropriate constant curvature background, and the resulting effective energy-momentum tensor is contracted with a background Killing vector, yielding a divergenceless vector current. The ADT energy is the associated conserved charge, which can be written as the flux of an antisymmetric tensor field (the superpotential) through a surface at spatial infinity. The results of [24] were applied in [15, 16] to compute the mass and angular momentum of the BTZ black holes in TMG. However they cannot be used to compute the corresponding charges for the ACL black holes, which are non-asymptotically AdS.

In this paper, we shall show how to compute the ADT charges for a solution of TMG linearized about an arbitrary background, and apply the result to evaluate the mass and angular momentum of ACL black holes. In Sect. 2 we describe briefly the general ADT procedure, and work out its application to TMG with an arbitrary background admitting a Killing vector field, resulting in the coordinate independent expression (2.22) for the associated ADT charge. In Sect. 3 we apply this to the computation of the mass and angular momentum of a generic stationary rotationally symmetric spacetime asymptotic to the as yet unspecified background. The application to the case of generalized ACL black holes is given in Sect. 4, leading to mass and angular momentum values which, together with the appropriate black hole entropy, are consistent with the first law. 


\section{Abbott-Deser-Tekin conserved charges for topo- logically massive gravity with arbitrary back- ground}

We first briefly summarize the ADT procedure for a theory of gravitation defined by the field equations

$$
\mathcal{E}_{\mu \nu}[g]=\kappa T_{\mu \nu}
$$

where $\mathcal{E}_{\mu \nu}[g]$ is a generalized Einstein tensor, $T_{\mu \nu}$ is the matter energymomentum tensor, and $\kappa=8 \pi G$ is the Einstein gravitational constant. Energy-momentum conservation implies that the left-hand side of (2.1) obeys the generalized Bianchi identities

$$
D_{\nu} \mathcal{E}^{\mu \nu}=0
$$

Consider a background metric $\bar{g}_{\mu \nu}$ solving the vacuum field equations

$$
\mathcal{E}_{\mu \nu}[\bar{g}]=0 .
$$

A generic metric $g_{\mu \nu}$, asymptotic to the background $\bar{g}_{\mu \nu}$, can be linearized around it as

$$
g_{\mu \nu}=\bar{g}_{\mu \nu}+\delta g_{\mu \nu} .
$$

By virtue of (2.2) and (2.3) , the linearized tensor $\delta \mathcal{E}_{\mu \nu}$ obeys the generalized Bianchi identities

$$
\bar{D}_{\nu} \delta \mathcal{E}^{\mu \nu}=0
$$

with $\bar{D}$ the background covariant derivative. Now if the background admits a Killing vector $\xi_{\mu}$, then the current

$$
\mathcal{K}^{\mu} \equiv \delta \mathcal{E}^{\mu \nu} \xi_{\nu}
$$

is covariantly conserved (as a consequence of (2.5) and the Killing equations):

$$
\bar{D}_{\mu} \mathcal{K}^{\mu}=\frac{1}{\sqrt{|\bar{g}|}} \partial_{\mu}\left(\sqrt{|\bar{g}|} \mathcal{K}^{\mu}\right)=0 .
$$

It follows that there exists an antisymmetric tensor field $\mathcal{F}^{\mu \nu}$ such that

$$
\mathcal{K}^{\mu}=\bar{D}_{\nu} \mathcal{F}^{\mu \nu},
$$

and that the charge

$$
Q(\xi)=\frac{1}{\kappa} \int_{M} \sqrt{|\bar{g}|} \mathcal{K}^{0}=\frac{1}{\kappa} \int_{\partial M} \sqrt{|\bar{g}|} \mathcal{F}^{0 i} d S_{i}
$$


does not depend on the space-like hypersurface $M$ of boundary $\partial M$.

Let us outline the application of this procedure to TMG with an arbitrary background (full details are given in Appendix A). To simplify the notation, we drop the bars on the background geometrical quantities, and write the linearized metric as

$$
\delta g_{\mu \nu} \equiv h_{\mu \nu} .
$$

Following [24, we write for TMG

$$
\delta \mathcal{E}_{\mu \nu} \equiv \delta \mathcal{G}_{\mu \nu}+\frac{1}{\mu} \delta C_{\mu \nu},
$$

with

$$
\mathcal{G}_{\mu \nu} \equiv G_{\mu \nu}-\Lambda g_{\mu \nu},
$$

and compute separately the Einstein and Cotton contributions to the current $\mathcal{K}^{\mu}$, neither of which is conserved alone.

The computation of the Einstein contribution follows closely [23], taking care however that, to the difference of the background in [23], $\mathcal{G}_{\mu \nu} \neq 0$ for our background. The result is 2

$$
\mathcal{K}_{E}^{\mu} \equiv \xi_{\nu} \delta \mathcal{G}^{\mu \nu}=D_{\lambda} \mathcal{F}_{E}^{\mu \lambda}(\xi)-\xi^{\nu} \mathcal{G}^{\mu \lambda} h_{\lambda \nu}+\frac{1}{2} \xi^{\mu} \mathcal{G}^{\lambda \rho} h_{\lambda \rho}-\frac{1}{2} \xi^{\nu} \mathcal{G}^{\mu}{ }_{\nu} h,
$$

where $h \equiv g^{\mu \nu} h_{\mu \nu}$, and $\mathcal{F}_{E}^{\mu \nu}(\xi)$ is the Einstein superpotential:

$$
\begin{aligned}
\mathcal{F}_{E}^{\mu \nu}(\xi)= & \frac{1}{2}\left[\xi^{\nu} D_{\lambda} h^{\lambda \mu}-\xi^{\mu} D_{\lambda} h^{\lambda \nu}+\xi_{\lambda} D^{\mu} h^{\lambda \nu}-\xi_{\lambda} D^{\nu} h^{\lambda \mu}+\xi^{\mu} D^{\nu} h-\xi^{\nu} D^{\mu} h\right. \\
& \left.+h^{\nu \lambda} D_{\lambda} \xi^{\mu}-h^{\mu \lambda} D_{\lambda} \xi^{\nu}+h D^{[\mu} \xi^{\nu]}\right] .
\end{aligned}
$$

To compute the Cotton contribution, we start as in 24] with the symmetric form of the Cotton tensor

$$
C^{\mu \nu}=\frac{1}{\sqrt{|g|}} \epsilon^{(\mu \alpha \beta} D_{\alpha} G_{\beta}^{\nu)},
$$

which is linearized to

$$
\delta C^{\mu \nu}=\frac{1}{\sqrt{|g|}} \epsilon^{(\mu \alpha \beta}\left(D_{\alpha} \delta G^{\nu)}{ }_{\beta}+\delta \Gamma_{\alpha \lambda}^{\nu)} G_{\beta}^{\lambda}\right)-\frac{1}{2} h C^{\mu \nu}
$$

\footnotetext{
${ }^{2}$ Note that $\delta \mathcal{G}^{\mu \nu}$ or $\delta \mathcal{G}^{\mu}{ }_{\nu}$ are the linearized $\mathcal{G}^{\mu \nu}$ or $\mathcal{G}^{\mu}{ }_{\nu}$, not to be confused with the contravariant or mixed components of $\delta \mathcal{G}_{\mu \nu}$.
} 
(the second and third terms on the r.h.s. vanish for the constant curvature background of [24]). This leads to

$$
\begin{aligned}
\mathcal{K}_{C}^{\mu} \equiv & \xi_{\nu} \delta C^{\mu \nu} \\
= & \frac{1}{2 \sqrt{|g|}}\left\{D_{\alpha}\left(\epsilon^{\mu \alpha \beta} \xi_{\nu} \delta G^{\nu}{ }_{\beta}+\epsilon^{\nu \alpha \beta} \xi_{\nu} \delta G^{\mu}{ }_{\beta}+\epsilon^{\mu \nu \beta} \xi_{\nu} \delta G^{\alpha}{ }_{\beta}\right)\right. \\
& -\epsilon^{\nu \alpha \beta} D_{\alpha} \xi_{\nu} \delta G^{\mu}{ }_{\beta}-\epsilon^{\mu \nu \beta} \xi_{\nu} D_{\alpha} \delta G^{\alpha}{ }_{\beta} \\
& \left.+2 \epsilon^{(\nu \alpha \beta} \xi_{\nu} \delta \Gamma_{\alpha \lambda}^{\mu)} G^{\lambda}{ }_{\beta}-\sqrt{|g|} \xi_{\nu} h C^{\mu \nu}\right\} .
\end{aligned}
$$

As in [24], the second term in the r.h.s. of (2.17) can be expressed in terms of the vector

$$
\eta^{\nu} \equiv \frac{1}{2 \sqrt{|g|}} \epsilon^{\nu \rho \sigma} D_{\rho} \xi_{\sigma}
$$

which obeys

$$
D^{\mu} \eta^{\nu}=\frac{1}{\sqrt{|g|}} \epsilon^{\mu \rho \lambda} \xi_{\rho} G_{\lambda}^{\nu},
$$

but is not a background Killing vector if the background is not constant curvature. The lengthy computation of (2.17), detailed in the Appendix, leads to the result

$$
\mathcal{K}_{C}^{\mu}=D_{\lambda} \mathcal{F}_{C}^{\mu \lambda}(\xi)-\xi^{\nu} C^{\lambda \mu} h_{\lambda \nu}+\frac{1}{2} \xi^{\mu} C^{\lambda \rho} h_{\lambda \rho}-\frac{1}{2} \xi^{\nu} C^{\mu}{ }_{\nu} h,
$$

with the Cotton superpotential

$$
\begin{aligned}
\mathcal{F}_{C}^{\mu \nu}(\xi) & =\mathcal{F}_{E}^{\mu \nu}(\eta)+\frac{1}{\sqrt{|g|}} \xi_{\lambda}\left(\epsilon^{\mu \nu \rho} \delta G_{\rho}^{\lambda}-\frac{1}{2} \epsilon^{\mu \nu \lambda} \delta G\right) \\
& +\frac{1}{2 \sqrt{|g|}} \epsilon^{\mu \nu \rho}\left[\xi_{\rho} h_{\sigma}^{\lambda} G_{\lambda}^{\sigma}+\frac{1}{2} h\left(\xi_{\sigma} G_{\rho}^{\sigma}+\frac{1}{2} \xi_{\rho} R\right)\right] .
\end{aligned}
$$

Taking into account the field equations (1.1) for the background, our final, coordinate invariant result for the ADT charge for TMG linearized about an arbitrary background with Killing vector $\xi$ is

$$
Q(\xi)=\frac{1}{\kappa} \int_{\partial M} \sqrt{|g|}\left(\mathcal{F}_{E}^{0 i}(\xi)+\frac{1}{\mu} \mathcal{F}_{C}^{0 i}(\xi)\right) d S_{i}
$$

where $\mathcal{F}_{E}^{\mu \nu}(\xi)$ and $\mathcal{F}_{C}^{\mu \nu}(\xi)$ are given in (2.14) and (2.21). 


\section{Application to stationary rotationally symmet- ric solutions}

We now specialize to spacetimes with two commuting Killing vectors (one time-like and the other space-like) $\partial_{t}$ and $\partial_{\varphi}$, and use the dimensional reduction procedure of [25, which we summarize here. We choose a coordinate system in such a way that the metric can be written as:

$$
d s^{2}=\lambda_{a b}(\rho) d x^{a} d x^{b}+\frac{1}{\zeta^{2} \mathcal{R}^{2}(\rho)} d \rho^{2},
$$

$\left(x^{0}=t, x^{1}=\varphi, x^{2}=\rho\right)$, where $\lambda$ is the $2 \times 2$ matrix

$$
\lambda=\left(\begin{array}{cc}
T+X & Y \\
Y & T-X
\end{array}\right),
$$

and $\zeta$ a constant scale factor. The vector $\mathbf{X}=(T, X, Y)$ belongs to a superspace endowed with a Minkowskian metric $\eta_{i j}(i=0,1,2)$ with "mostly plus" signature, the norm being defined by

$$
\mathcal{R}^{2} \equiv \mathbf{X}^{2}=\eta_{i j} X^{i} X^{j}=-T^{2}+X^{2}+Y^{2} .
$$

It is natural to define the dot product of two supervectors $\mathbf{A}$ and $\mathbf{B}$ by their Minkowskian scalar product, and their wedge product by

$$
(\mathbf{A} \wedge \mathbf{B})^{i}=\eta^{i j} \epsilon_{j k l} A^{k} B^{l},
$$

where $\epsilon_{i j k}$ is the Levi-Cevita symbol, with $\epsilon_{012}=+1$ (thus $\epsilon^{012}=-1$ ).

To each supervector $\mathbf{A}$, we associate a traceless matrix noted $[\mathbf{A}]$ or $a$ such that

$$
a \equiv[\mathbf{A}] \equiv \boldsymbol{\tau} \cdot \mathbf{A}=\left(\begin{array}{cc}
-A^{Y} & -A^{-} \\
A^{+} & A^{Y}
\end{array}\right)
$$

where

$$
A^{ \pm} \equiv A^{T} \pm A^{X}
$$

and $\tau^{i}$ are the (real) Pauli matrices

$$
\tau^{0}=\left(\begin{array}{cc}
0 & 1 \\
-1 & 0
\end{array}\right), \tau^{1}=\left(\begin{array}{ll}
0 & 1 \\
1 & 0
\end{array}\right), \tau^{2}=\left(\begin{array}{cc}
-1 & 0 \\
0 & 1
\end{array}\right)
$$

satisfying

$$
\tau^{i} \tau^{j}=\eta^{i j}+\epsilon^{i j k} \tau_{k}, \quad\left(\tau^{i}\right)^{t}=\tau^{0} \tau^{i} \tau^{0}=\tau_{i} .
$$


It follows immediately from the above two properties that for any two supervectors $\mathbf{A}, \mathbf{B}$ one has

$$
[\mathbf{A}][\mathbf{B}]=(\mathbf{A} \cdot \mathbf{B}) \underline{1}+[\mathbf{A} \wedge \mathbf{B}]
$$

where 1 is the $2 \times 2$ unit matrix.

Let us apply this formalism to the computation of the ADT charges (2.22). Choosing the boundary $\partial M$ to be a circle, we need only to compute the (02) superpotential components. This computation, outlined in Appendix B, leads to

$$
\begin{aligned}
& \mathcal{F}_{E}^{02}(\xi)=\frac{1}{2} \zeta^{2}\left[\left(-\delta[\mathbf{L}]+\mathbf{X} \cdot \delta \mathbf{X}^{\prime}\right) \xi\right]^{0} \\
& \left.\mathcal{F}_{C}^{02}(\xi)=\frac{1}{2} \zeta^{3}\left\{\left(\delta\left[\mathbf{X} \wedge \mathbf{L}^{\prime}\right]-\frac{1}{2} \delta\left[\mathbf{X}^{\prime} \wedge \mathbf{L}\right]+\frac{1}{2} \mathbf{X}^{\prime} \cdot \delta \mathbf{L}-\mathbf{X} \cdot \delta \mathbf{L}^{\prime}\right) \xi\right\}^{0} 3.11\right)
\end{aligned}
$$

The net ADT charge (2.22) is a linear combination of the Einstein superpotential (3.10) and the Cotton superpotential (3.11). To make contact with the SAM approach of [4], we define the spin super angular momentum

$$
\mathbf{S} \equiv \frac{\zeta}{\mu}\left[\frac{1}{2} \mathbf{X}^{\prime} \wedge \mathbf{L}-\mathbf{X} \wedge \mathbf{L}^{\prime}\right]
$$

and the total conserved super angular momentum [26]

$$
\mathbf{J}=\mathbf{L}+\mathbf{S},
$$

in terms of which the Killing charge is given by

$$
Q(\xi)=-\frac{\pi \zeta}{\kappa}\left\{\left(\delta[\mathbf{J}]-\mathbf{X} \cdot \delta \mathbf{X}^{\prime}+\frac{\zeta}{\mu}\left(\mathbf{X} \cdot \delta \mathbf{L}^{\prime}-\frac{1}{2} \mathbf{X}^{\prime} \cdot \delta \mathbf{L}\right)\right) \xi\right\}^{0} .
$$

Choosing $\xi$ to be one of the two Killing vectors of (3.1), $\xi_{(t)}=(-1,0)$ and $\xi_{(\varphi)}=(0,1)$, we finally obtain the mass and angular momentum of the field configuration $\mathbf{X}+\delta \mathbf{X}$ relative to the background $\mathbf{X}$ :

$$
\begin{aligned}
\mathcal{M} & =-\frac{\pi \zeta}{\kappa}\left[\delta J^{Y}+\mathbf{X} \cdot \delta \mathbf{X}^{\prime}+\frac{\zeta}{\mu}\left(\frac{1}{2} \mathbf{X}^{\prime} \cdot \delta \mathbf{L}-\mathbf{X} \cdot \delta \mathbf{L}^{\prime}\right)\right] \\
\mathcal{J} & =\frac{\pi \zeta}{\kappa} \delta J^{-} .
\end{aligned}
$$

We recall that the super angular momentum associated with a solution of the field equations (1.1) is constant (independent of $\rho$ ). If the background is 
chosen so that the components $J^{Y}$ and $J^{-}$of its super angular momentum vanish, then $\delta J^{Y}$ and $\delta J^{-}$are the corresponding super angular momentum components of the self-gravitating configuration under consideration. Comparing with Eqs (16) and (17) of [4], we see 3 that the ADT angular momentum is identical with the SAM expression, while the ADT mass differs from the SAM mass $-(\pi \zeta / \kappa) J^{Y}$ by the last three terms of (3.15).

To conclude this section, we note that the ADT corrections to the SAM mass vanish in the case of the BTZ black holes. The reason is that the BTZ solution is of the form

$$
\mathbf{X}=\boldsymbol{\alpha} \rho+\boldsymbol{\beta},
$$

where the constant supervector $\boldsymbol{\alpha}$ is universal (the same for all BTZ black holes), and the constant supervector $\boldsymbol{\beta}$ depends on the black hole mass and angular momentum. It follows from (3.17) that $\mathbf{X}^{\prime}=\boldsymbol{\alpha}$, so that $\delta \mathbf{X}^{\prime}=0$, and $\delta \mathbf{L}=\delta \mathbf{X} \wedge \mathbf{X}^{\prime}$ leading to $\mathbf{X}^{\prime} \cdot \delta \mathbf{L}=0$ and $\delta \mathbf{L}^{\prime}=0$.

\section{The TMG black holes: mass, angular momen- tum, and the first law}

The ACL black hole solution of the TMG field equations (1.1) is [27]

$$
\begin{aligned}
& d s^{2}=-\beta^{2} \frac{\rho^{2}-\rho_{0}^{2}}{r^{2}} d t^{2}+\frac{1}{\zeta^{2} \beta^{2}} \frac{d \rho^{2}}{\rho^{2}-\rho_{0}^{2}} \\
&+r^{2}\left[d \varphi-\frac{\rho+\left(1-\beta^{2}\right) \omega}{r^{2}} d t\right]^{2}
\end{aligned}
$$

with

$$
r^{2}=\rho^{2}+2 \omega \rho+\omega^{2}\left(1-\beta^{2}\right)+\frac{\beta^{2} \rho_{0}^{2}}{1-\beta^{2}},
$$

where

$$
\beta^{2} \equiv \frac{1}{4}\left(1-\frac{27 \Lambda}{\mu^{2}}\right)
$$

we have chosen

$$
\zeta=\frac{2}{3} \mu
$$

and the two parameters $\omega$ and $\rho_{0} \geq 0$ are related to the black hole mass and angular momentum. This solution is related to the solutions with horizons given by Nutku [5] and Gürses [6] by the double Wick rotation $t \rightarrow i t$, $\varphi \rightarrow i \varphi$. For $\Lambda=0\left(\beta^{2}=1 / 4\right)$ and $\mu=3$, the black hole metric reduces to

\footnotetext{
${ }^{3}$ The $\mathbf{J}$ in 4 should be multiplied by $\zeta / 2 \kappa$ to conform to our present convention.
} 
that of the ACL black hole [4] after rescaling $t \rightarrow 2 t$ and $\omega \rightarrow 2 \omega$. If $\rho_{0}>0$, the two horizons of (4.1) are located at $\rho= \pm \rho_{0}$. As will be shown in [27], these black holes are causally regular and geodesically complete (except in the case $\omega=\rho_{0} /\left(1-\beta^{2}\right)$, where the inner horizon $\rho=-\rho_{0}$ becomes singular) if $0<\beta^{2}<1$ and $\omega>-\rho_{0} / \sqrt{1-\beta^{2}}$. If $\rho_{0}=0$ the black hole becomes extreme with a double horizon at $\rho=0$, except in the special case $\omega=\rho_{0}=0$, where the metric (4.1) reduces to

$$
d s^{2}=-\beta^{2} d t^{2}+\frac{1}{\zeta^{2} \beta^{2}} \frac{d \rho^{2}}{\rho^{2}}+\rho^{2}\left[d \varphi-\frac{d t}{\rho}\right]^{2},
$$

which is horizonless, and thus qualifies as the ground state or "vacuum" of the black-hole family (4.1).

\subsection{Mass and angular momentum}

The vector $\mathbf{X}$ associated with (4.1) is of the form 4

$$
\mathbf{X}(\rho)=\boldsymbol{\alpha} \rho^{2}+\boldsymbol{\beta} \rho+\gamma,
$$

where

$$
\boldsymbol{\alpha}=(1 / 2,-1 / 2,0), \quad \boldsymbol{\beta}=(\omega,-\omega,-1), \quad \boldsymbol{\gamma}=(z+u, z-u,-2 \omega z),
$$

and

$$
z=\frac{1-\beta^{2}}{2} \quad u=\omega^{2} z+\frac{\beta^{2} \rho_{0}^{2}}{4 z} .
$$

From the wedge products

$$
\boldsymbol{\alpha} \wedge \boldsymbol{\beta}=-\boldsymbol{\alpha}, \quad \boldsymbol{\alpha} \wedge \boldsymbol{\gamma}=-z \boldsymbol{\beta}, \quad \boldsymbol{\beta} \wedge \gamma=\frac{\beta^{2} \rho^{2}}{z} \boldsymbol{\alpha}-\gamma
$$

we obtain

$$
\begin{aligned}
& \mathbf{L}=\boldsymbol{\alpha} \rho^{2}+2 \boldsymbol{\beta} z \rho-\boldsymbol{\beta} \wedge \boldsymbol{\gamma} \\
& \mathbf{S}=-\boldsymbol{\alpha} \rho^{2}-2 \boldsymbol{\beta} z \rho-\frac{1}{3} \boldsymbol{\gamma}+\frac{4}{3} z \boldsymbol{\beta} \wedge \boldsymbol{\gamma}
\end{aligned}
$$

leading to the constant super angular momentum

$$
\mathbf{J}=\frac{2 \beta^{2}}{3}\left[-\frac{1+2 \beta^{2}}{1-\beta^{2}} \rho_{0}^{2} \boldsymbol{\alpha}+\gamma\right] .
$$

\footnotetext{
${ }^{4}$ In this section we return to the convention introduced in Eq. (2.4), i.e. background geometrical quantities are overlined.
} 
The components $J^{Y}$ and $J^{-}$vanish for the background (4.4), so that the $\mathrm{SAM}$ mass $\mathcal{M}_{0}$ and the angular momentum $\mathcal{J}$ are

$$
\begin{aligned}
\mathcal{M}_{0} & =-\frac{\pi \zeta}{\kappa} J^{Y}=\frac{4 \pi \mu}{9 \kappa} \beta^{2}\left(1-\beta^{2}\right) \omega, \\
\mathcal{J} & =\frac{\pi \zeta}{\kappa} J^{-}=\frac{4 \pi \mu}{9 \kappa} \beta^{2}\left[\left(1-\beta^{2}\right) \omega^{2}-\frac{1+\beta^{2}}{1-\beta^{2}} \rho_{0}^{2}\right] .
\end{aligned}
$$

Next we compute the correction $\Delta \mathcal{M}$ to the SAM mass coming from the last three terms of (3.15). The constant vectors (4.6) associated with the background (4.4) and the deviations from the background are

$$
\begin{aligned}
\overline{\boldsymbol{\alpha}} & =\boldsymbol{\alpha}, \quad \overline{\boldsymbol{\beta}}=(0,0,-1), \quad \overline{\boldsymbol{\gamma}}=(z, z, 0), \\
\delta \boldsymbol{\alpha} & =0, \quad \delta \boldsymbol{\beta}=2 \omega \boldsymbol{\alpha}, \quad \delta \boldsymbol{\gamma}=2 u \boldsymbol{\alpha}+2 \omega z \overline{\boldsymbol{\beta}},
\end{aligned}
$$

with the only non-vanishing scalar products

$$
\overline{\boldsymbol{\beta}} \cdot \delta \boldsymbol{\gamma}=-\overline{\boldsymbol{\gamma}} \cdot \delta \boldsymbol{\beta}=2 \omega z, \quad \overline{\boldsymbol{\gamma}} \cdot \delta \boldsymbol{\gamma}=-2 \omega^{2} z^{2}-\frac{\beta^{2} \rho_{0}^{2}}{2}
$$

This leads to

$$
\Delta \mathcal{M}=\frac{4 \pi \mu}{9 \kappa} \beta^{2}\left(1-\beta^{2}\right) \omega=\mathcal{M}_{0} .
$$

Thus the ADT mass for the black hole (4.1) is twice the SAM mass:

$$
\mathcal{M}=\frac{8 \pi \mu}{9 \kappa} \beta^{2}\left(1-\beta^{2}\right) \omega .
$$

\subsection{The first law}

Now we check that these values for the black hole mass and angular momentum are consistent with the first law of black hole thermodynamics.

$$
d \mathcal{M}=T_{H} d S+\Omega_{h} d \mathcal{J} .
$$

From the black hole metric (4.1), which is already in the ADM form

$$
d s^{2}=-N^{2} d t^{2}+r^{2}\left(d \varphi+N^{\varphi} d t\right)^{2}+\frac{1}{(\zeta r N)^{2}} d \rho^{2}
$$

we read the Hawking temperature

$$
T_{H}=\left.\frac{1}{2 \pi} n^{\rho} \partial_{\rho} N\right|_{\rho=\rho_{0}}=\frac{\mu \beta^{2}}{3 \pi} \frac{\rho_{0}}{r_{h}},
$$


where $n^{\rho}=\sqrt{g^{\rho \rho}}=\zeta r N$, and

$$
r_{h}=r\left(\rho_{0}\right)=\frac{1}{\sqrt{1-\beta^{2}}}\left[\rho_{0}+\left(1-\beta^{2}\right) \omega\right]
$$

is the horizon areal radius. The horizon angular velocity is

$$
\Omega_{h}=-N^{\varphi}\left(\rho_{0}\right)=\frac{\sqrt{1-\beta^{2}}}{r_{h}} .
$$

The final ingredient is the black hole entropy $S$. This is the sum

$$
S=S_{E}+S_{C}
$$

of an Einstein and a Cotton (or Chern-Simons) contribution. The Einstein entropy is as usual proportional to the horizon "area" (perimeter in the present case),

$$
S_{E}=\frac{4 \pi^{2}}{\kappa} r_{h} .
$$

The general formula for the Chern-Simons contribution to the entropy was first given by Solodukhin [18], and rederived by Tachikawa [28]. This is

$$
S_{C}=-\frac{2 \pi}{\kappa \mu} \int_{0}^{2 \pi} \omega_{02, \varphi} d \varphi
$$

evaluated on the horizon, where $\omega_{a b}=\omega_{a b, \mu} d x^{\mu}$ is the spin connection. The dreibein $e^{a}$ for the metric (4.19) is

$$
e^{0}=N d t, \quad e^{1}=r\left(d \varphi+N^{\varphi}\right) d t, \quad e^{2}=\frac{1}{\zeta r N} d \rho .
$$

The corresponding spin connections are

$$
\begin{aligned}
\omega_{2}^{0} & =\zeta r\left[N^{\prime} e^{0}+\frac{1}{2} r\left(N^{\varphi}\right)^{\prime} e^{1}\right], \\
\omega^{0}{ }_{1} & =\zeta r \frac{1}{2} r\left(N^{\varphi}\right)^{\prime} e^{2}, \\
\omega^{1} & =\zeta r\left[\frac{1}{2} r\left(N^{\varphi}\right)^{\prime} e^{0}+N \frac{r^{\prime}}{r} e^{1}\right],
\end{aligned}
$$

leading to

$$
S_{C}=-\frac{2 \pi^{2}}{\kappa \mu} \zeta r_{h}^{3}\left(N^{\varphi}\right)^{\prime}\left(\rho_{0}\right)=-\frac{4 \pi^{2}}{3 \kappa \sqrt{1-\beta^{2}}}\left[\left(1-2 \beta^{2}\right) \rho_{0}+\left(1-\beta^{2}\right) \omega\right] .
$$


The total entropy is

$$
S=\frac{8 \pi^{2}}{3 \kappa \sqrt{1-\beta^{2}}}\left[\left(1+\beta^{2}\right) \rho_{0}+\left(1-\beta^{2}\right) \omega\right] .
$$

Putting (4.17), (4.13) and (4.29) together, it is easy to check that the first law (4.18) is satisfied for independent variations of the black hole parameters $\rho_{0}$ and $\omega$.

\section{Conclusion}

We have shown that the Abbott-Deser-Tekin approach can be extended to the computation of the mass and angular momentum of solutions of topologically massive gravity asymptotic to an arbitrary, non constant curvature, background. In the case of the ACL black holes of cosmological TMG, the resulting mass and angular momentum, together with the SolodukhinTachikawa entropy, fit nicely into the first law of black hole thermodynamics. It would be interesting to extend our work in order to compute the mass and angular momentum of the black hole solutions to topologically massive gravitoelectrodynamics [27, as well as of black hole solutions with non-constant curvature asymptotics in the framework of other theories of gravity. The

tedious computations involved could perhaps be streamlined by following the procedure advocated in [29].

\section{Appendix A: Computation of the Einstein and Cot- ton superpotentials}

Our conventions for the Riemann and Ricci tensors are:

$$
\left[D_{\lambda}, D_{\nu}\right] \xi^{\mu}=R_{\rho \lambda \nu}^{\mu} \xi^{\rho}, \quad R_{\mu \lambda \nu}^{\lambda}=R_{\mu \nu}
$$

and the Einstein tensors are defined by

$$
G_{\mu \nu} \equiv R_{\mu \nu}-\frac{1}{2} R g_{\mu \nu}, \quad \mathcal{G}_{\mu \nu} \equiv G_{\mu \nu}+\mathrm{Ł} g_{\mu \nu} .
$$

From the linearized metric $\delta g_{\mu \nu} \equiv h_{\mu \nu}$, we obtain the linearized Christoffels

$$
\delta \Gamma_{\mu \nu}^{\rho}=\frac{1}{2}\left(D_{\mu} h_{\nu}^{\rho}+D_{\nu} h_{\mu}^{\rho}-D^{\rho} h_{\mu \nu}\right)
$$


and the linearized Ricci tensor

$$
\begin{aligned}
\delta R_{\mu \nu} & =D_{\rho} \delta \Gamma_{\mu \nu}^{\rho}-D_{\nu} \delta \Gamma_{\mu \rho}^{\rho} \\
& =\frac{1}{2}\left(D^{\lambda} D_{\nu} h_{\lambda \mu}+D^{\lambda} D_{\mu} h_{\lambda \nu}-D^{\lambda} D_{\lambda} h_{\mu \nu}-D_{\mu} D_{\nu} h\right),
\end{aligned}
$$

with $h \equiv g^{\mu \nu} h_{\mu \nu}$.

The computation of the Killing current $\mathcal{K}_{E}^{\mu} \equiv \xi_{\nu} \delta \mathcal{G}^{\mu \nu}$ by successive integration by parts follows closely [23]:

$$
\begin{aligned}
2 \mathcal{K}_{E}^{\mu}= & \xi_{\nu}\left(D_{\lambda} D^{\nu} h^{\lambda \mu}+D_{\lambda} D^{\mu} h^{\lambda \nu}-D^{\lambda} D_{\lambda} h^{\mu \nu}-D^{\mu} D^{\nu} h\right) \\
& +\xi^{\mu}\left(D_{\lambda} D^{\lambda} h-D_{\lambda} D_{\nu} h^{\lambda \nu}\right)+\xi_{\nu}\left(-4 R^{(\mu \lambda} h_{\lambda}{ }^{\nu)}+(R-2 \mathrm{E}) h^{\mu \nu}\right) \\
& +\xi^{\mu} R_{\lambda \rho} h^{\lambda \rho} \\
= & D_{\lambda}\left(\xi^{\lambda} D_{\nu} h^{\mu \nu}-\xi^{\mu} D_{\nu} h^{\lambda \nu}+\xi_{\nu} D^{\mu} h^{\lambda \nu}-\xi_{\nu} D^{\lambda} h^{\mu \nu}+\xi^{\mu} D^{\lambda} h-\xi^{\lambda} D^{\mu} h\right) \\
& +D_{\lambda} \xi_{\nu} D^{\lambda} h^{\mu \nu}+D_{\lambda} \xi^{\mu} D_{\nu} h^{\lambda \nu}-D_{\lambda} \xi^{\mu} D^{\lambda} h \\
& +\xi^{\nu}\left(-2 R^{\mu \lambda} h_{\lambda \nu}-R_{\nu \lambda} h^{\lambda \mu}+R^{\mu}{ }_{\rho \lambda \nu} h^{\lambda \rho}+(R-2 \mathrm{E}) h^{\mu \nu}\right) \\
& +\xi^{\mu} R_{\lambda \rho} h^{\lambda \rho} \\
= & 2 D_{\lambda} \mathcal{F}_{E}^{\mu \lambda}(\xi)-2 \xi^{\nu} \mathcal{G}^{\lambda \mu} h_{\lambda \nu}+\xi^{\mu} \mathcal{G}^{\lambda \rho} h_{\lambda \rho}-\xi^{\nu} \mathcal{G}^{\mu}{ }_{\nu} h,
\end{aligned}
$$

where the Einstein superpotential is:

$$
\begin{aligned}
2 \mathcal{F}_{E}^{\mu \nu}(\xi)= & \xi^{\nu} D_{\lambda} h^{\lambda \mu}-\xi^{\mu} D_{\lambda} h^{\lambda \nu}+\xi_{\lambda} D^{\mu} h^{\lambda \nu}-\xi_{\lambda} D^{\nu} h^{\lambda \mu}+\xi^{\mu} D^{\nu} h-\xi^{\nu} D^{\mu} h \\
& +h^{\nu \lambda} D_{\lambda} \xi^{\mu}-h^{\mu \lambda} D_{\lambda} \xi^{\nu}+h D^{[\mu} \xi^{\nu]}
\end{aligned}
$$

and we have used the identity valid for Killing vectors

$$
D_{\mu} D_{\nu} \xi_{\lambda}=R_{\mu \nu \lambda}^{\rho} \xi_{\rho}
$$

From the Cotton tensor

$$
C^{\mu \nu}=\frac{1}{\sqrt{|g|}} \epsilon^{(\mu \alpha \beta} D_{\alpha} G_{\beta}^{\nu)},
$$

with $\epsilon^{\mu \alpha \beta}$ the antisymmetric symbol, we obtain the linearized Cotton tensor

$$
\delta C^{\mu \nu}=\frac{1}{\sqrt{|g|}} \epsilon^{(\mu \alpha \beta}\left(D_{\alpha} \delta G^{\nu)}{ }_{\beta}+\delta \Gamma_{\alpha \lambda}^{\nu)} G_{\beta}^{\lambda}\right)-\frac{1}{2} h C^{\mu \nu} .
$$


The resulting Killing current $\mathcal{K}_{C}^{\mu} \equiv \xi_{\nu} \delta C^{\mu \nu}$ is

$$
\begin{aligned}
2 \sqrt{|g|} \mathcal{K}_{C}^{\mu}= & D_{\alpha}\left(\epsilon^{\mu \alpha \beta} \xi_{\nu} \delta G^{\nu}{ }_{\beta}+\epsilon^{\nu \alpha \beta} \xi_{\nu} \delta G^{\mu}{ }_{\beta}+\epsilon^{\mu \nu \beta} \xi_{\nu} \delta G^{\alpha}{ }_{\beta}\right) \\
& -\epsilon^{\nu \alpha \beta} D_{\alpha} \xi_{\nu} \delta G^{\mu}{ }_{\beta}-\epsilon^{\mu \nu \beta} \xi_{\nu} D_{\alpha} \delta G^{\alpha}{ }_{\beta} \\
& +2 \epsilon^{(\nu \alpha \beta} \xi_{\nu} \delta \Gamma_{\alpha \lambda}^{\mu)} G^{\lambda}{ }_{\beta}-\sqrt{|g|} \xi_{\nu} h C^{\mu \nu} .
\end{aligned}
$$

Using

$$
D_{\alpha} \delta G^{\alpha}{ }_{\beta}=-\delta \Gamma_{\alpha \lambda}^{\alpha} G_{\beta}^{\lambda}+\delta \Gamma_{\alpha \beta}^{\lambda} G_{\lambda}^{\alpha},
$$

and the identity

$$
\epsilon^{\mu \alpha \beta} \xi_{\nu} \equiv \delta_{\nu}^{\mu} \epsilon^{\alpha \beta \rho} \xi_{\rho}+\delta_{\nu}^{\alpha} \epsilon^{\beta \mu \rho} \xi_{\rho}+\delta_{\nu}^{\beta} \epsilon^{\mu \alpha \rho} \xi_{\rho}
$$

we obtain

$$
\begin{aligned}
X^{\mu} \equiv & \sqrt{|g|}\left(2 \mathcal{K}_{C}^{\mu}+2 \xi^{\nu} C^{\lambda \mu} h_{\lambda \nu}-\xi^{\mu} C^{\lambda \rho} h_{\lambda \rho}+\xi^{\nu} C^{\mu}{ }_{\nu} h\right) \\
= & D_{\alpha}\left(2 \epsilon^{\mu \alpha \beta} \xi_{\nu} \delta G^{\nu}{ }_{\beta}-\epsilon^{\mu \alpha \nu} \xi_{\nu} \delta G\right)-\epsilon^{\nu \alpha \beta} D_{\alpha} \xi_{\nu} \delta G^{\mu}{ }_{\beta} \\
& +2\left(\epsilon^{\alpha \beta \nu} \delta \Gamma_{\alpha \lambda}^{\mu}+\epsilon^{\mu \alpha \nu} \delta \Gamma_{\alpha \lambda}^{\beta}+\epsilon^{\beta \mu \nu} \delta \Gamma_{\alpha \lambda}^{\alpha}\right) \xi_{\nu} G^{\lambda}{ }_{\beta} \\
& +\left(\epsilon^{\lambda \alpha \beta} \xi_{\nu} D_{\alpha} G^{\mu}{ }_{\beta}+\epsilon^{\mu \alpha \beta} \xi_{\nu} D_{\alpha} G^{\lambda}{ }_{\beta}-\epsilon^{\lambda \alpha \beta} \xi^{\mu} D_{\alpha} G_{\nu \beta}\right) h_{\lambda}{ }^{\nu}(
\end{aligned}
$$

Defining the vector

$$
\eta^{\nu} \equiv \frac{1}{2 \sqrt{|g|}} \epsilon^{\nu \rho \sigma} D_{\rho} \xi_{\sigma}
$$

and expressing the 3-dimensional Riemann tensor in terms of the Einstein tensor,

$$
R_{\mu \rho \lambda \nu}=g_{\mu \lambda} G_{\rho \nu}-g_{\mu \nu} G_{\rho \lambda}+g_{\rho \nu} G_{\mu \lambda}-g_{\rho \lambda} G_{\mu \nu}+\frac{1}{2}\left(g_{\mu \lambda} g_{\rho \nu}-g_{\mu \nu} g_{\rho \lambda}\right) R,
$$

we have from (A.9) and (A.14)

$$
D^{\mu} \eta^{\nu}=\frac{1}{\sqrt{|g|}} \epsilon^{\mu \rho \lambda} \xi_{\rho} G_{\lambda}^{\nu} .
$$

Using the definition (A.17), the second term of (A.16) can be rewritten as

$$
-\epsilon^{\nu \alpha \beta} D_{\alpha} \xi_{\nu} \delta G^{\mu}{ }_{\beta}=2 \sqrt{|g|} \eta^{\beta} \delta G_{\beta}^{\mu}
$$




$$
\begin{aligned}
& =\sqrt{|g|} D_{\lambda}\left(\eta^{\lambda} D_{\nu} h^{\mu \nu}-\eta^{\mu} D_{\nu} h^{\lambda \nu}+\eta_{\nu} D^{\mu} h^{\lambda \nu}-\eta_{\nu} D^{\lambda} h^{\mu \nu}+\eta^{\mu} D^{\lambda} h-\eta^{\lambda} D^{\mu} h\right) \\
& +\sqrt{|g|}\left(D_{\lambda} \eta^{\mu} D_{\nu} h^{\lambda \nu}-D_{\lambda} \eta_{\nu} D^{\mu} h^{\lambda \nu}+D_{\lambda} \eta_{\nu} D^{\lambda} h^{\mu \nu}-D_{\lambda} \eta^{\mu} D^{\lambda} h\right) \\
& +\sqrt{|g|} \eta^{\beta}\left(h^{\lambda}{ }_{\beta} G^{\mu}{ }_{\lambda}-h G^{\mu}{ }_{\beta}\right),
\end{aligned}
$$

while the third term can be rewritten as

$$
\begin{aligned}
& 2\left(\epsilon^{\alpha \beta \nu} \delta \Gamma_{\alpha \lambda}^{\mu}+\epsilon^{\mu \alpha \nu} \delta \Gamma_{\alpha \lambda}^{\beta}+\epsilon^{\beta \mu \nu} \delta \Gamma_{\alpha \lambda}^{\alpha}\right) \xi_{\nu} G_{\beta}^{\lambda}= \\
& =\sqrt{|g|} D^{\alpha} \eta^{\lambda}\left(-D_{\alpha} h^{\mu}{ }_{\lambda}-D_{\lambda} h^{\mu}{ }_{\alpha}+D^{\mu} h_{\alpha \lambda}+\delta_{\alpha}^{\mu} D_{\lambda} h\right) \\
& +\epsilon^{\mu \alpha \nu} \xi_{\nu} G^{\lambda}{ }_{\beta} D_{\alpha} h_{\lambda}^{\beta} .
\end{aligned}
$$

Collecting these, and again integrating by parts, we arrive at

$$
\begin{aligned}
X^{\mu}= & D_{\lambda}\left[2 \epsilon^{\mu \lambda \beta} \xi_{\nu} \delta G^{\nu}{ }_{\beta}-\epsilon^{\mu \lambda \nu} \xi_{\nu} \delta G+\sqrt{|g|}\left(\eta^{\lambda} D_{\nu} h^{\mu \nu}-\eta^{\mu} D_{\nu} h^{\lambda \nu}\right.\right. \\
& +\eta_{\nu} D^{\mu} h^{\lambda \nu}-\eta_{\nu} D^{\lambda} h^{\mu \nu}+\eta^{\mu} D^{\lambda} h-\eta^{\lambda} D^{\mu} h+D_{\nu} \eta^{\mu} h^{\lambda \nu} \\
& \left.\left.-D_{\nu} \eta^{\mu} h^{\lambda \nu}+D^{\mu} \eta^{\lambda} h-D^{\lambda} \eta^{\mu} h\right)+\epsilon^{\mu \lambda \nu} \xi_{\nu} G^{\rho}{ }_{\beta} h^{\beta}{ }_{\rho}\right] \\
& -2 \sqrt{|g|}\left(D_{\lambda} D_{\nu} \eta^{[\mu} h^{\lambda] \nu}+D_{\lambda} D^{[\mu} \eta^{\lambda]} h\right) \\
& -\epsilon^{\mu \lambda \nu} D_{\lambda}\left(\xi_{\nu} G^{\rho}{ }_{\beta}\right) h_{\rho}^{\beta}+\sqrt{|g|} \eta^{\beta}\left(h^{\lambda}{ }_{\beta} G^{\mu}{ }_{\lambda}-h G^{\mu}{ }_{\beta}\right) \\
& +\left(\epsilon^{\lambda \alpha \beta} \xi_{\nu} D_{\alpha} G^{\mu}{ }_{\beta}+\epsilon^{\mu \alpha \beta} \xi_{\nu} D_{\alpha} G^{\lambda}{ }_{\beta}-\epsilon^{\lambda \alpha \beta} \xi^{\mu} D_{\alpha} G_{\nu \beta}\right) h_{\lambda}{ }^{\nu} . \text { A. }
\end{aligned}
$$

Using (A.17) and (A.19), and the identity (obtained [30] by computing $\left[D^{\lambda}, D^{\nu}\right] \eta^{\mu}$, first from (A.1), then from (A.19) $)$

$$
\begin{aligned}
& \epsilon^{\rho \nu \sigma} \xi_{\sigma} D^{\lambda} G^{\mu}{ }_{\rho}-\epsilon^{\rho \lambda \sigma} \xi_{\sigma} D^{\nu} G_{\rho}^{\mu}= \\
& \quad=\sqrt{|g|}\left(g^{\mu \lambda} G^{\tau \nu} \eta_{\tau}-g^{\mu \nu} G^{\tau \lambda} \eta_{\tau}+2 G^{\mu \lambda} \eta^{\nu}-2 G^{\mu \nu} \eta^{\lambda}\right)
\end{aligned}
$$

we finally obtain

$$
2 \mathcal{K}_{C}^{\mu}=2 D_{\lambda} \mathcal{F}_{C}^{\mu \lambda}(\xi)-2 \xi^{\nu} C^{\lambda \mu} h_{\lambda \nu}+\xi^{\mu} C^{\lambda \rho} h_{\lambda \rho}-\xi^{\nu} C^{\mu}{ }_{\nu} h
$$

with the Cotton superpotential:

$$
\begin{aligned}
2 \mathcal{F}_{C}^{\mu \nu}(\xi) & =2 \mathcal{F}_{E}^{\mu \nu}(\eta)+\frac{1}{\sqrt{|g|}} \xi_{\lambda}\left(2 \epsilon^{\mu \nu \rho} \delta G_{\rho}^{\lambda}-\epsilon^{\mu \nu \lambda} \delta G\right) \\
& +\frac{1}{\sqrt{|g|}} \epsilon^{\mu \nu \rho}\left[\xi_{\rho} h_{\sigma}^{\lambda} G_{\lambda}^{\sigma}+\frac{1}{2} h\left(\xi_{\sigma} G_{\rho}^{\sigma}+\frac{1}{2} \xi_{\rho} R\right)\right] .
\end{aligned}
$$




\section{Appendix B: Computation of the ADT charges of stationary rotationally symmetric solutions}

From the background metric (3.1), we compute the Christoffel symbols

$$
\Gamma_{2 b}^{a}=\frac{1}{2}\left(\lambda^{-1} \lambda^{\prime}\right)^{a}{ }_{b}, \quad \Gamma_{a b}^{2}=-\frac{1}{2} \zeta^{2} \mathcal{R}^{2} \lambda^{\prime}{ }_{a b}, \quad \Gamma_{22}^{2}=-\mathcal{R}^{-1} \mathcal{R}^{\prime},
$$

where the prime stands for the derivative $d / d \rho$. The matrices in (B.1) are related to the matrix $x$ (defined according to (3.5)) by

$$
\lambda=\tau^{0} x, \quad \lambda^{-1}=-\frac{1}{\mathcal{R}^{2}} x \tau^{0} .
$$

This leads to the Ricci tensor components

$$
R_{b}^{a}=-\frac{\zeta^{2}}{2}\left(\left(\mathcal{R} \mathcal{R}^{\prime}\right)^{\prime} \mathbf{1}+\ell^{\prime}\right)_{b}^{a}, \quad R_{2}^{2}=\zeta^{2}\left(-\left(\mathcal{R} \mathcal{R}^{\prime}\right)^{\prime}+\frac{1}{2} \mathbf{X}^{\prime 2}\right),
$$

where $\ell$ is the matrix associated with the vector

$$
\mathbf{L} \equiv \mathbf{X} \wedge \mathbf{X}^{\prime}
$$

The linearized metric components are

$$
h_{a b}=\delta \lambda_{a b}, \quad h_{22}=-2 \zeta^{-2} \frac{\delta \mathcal{R}}{\mathcal{R}^{3}} .
$$

We will also need the mixed components

$$
h_{b}^{a}=\left(\lambda^{-1} \delta \lambda\right)^{a}{ }_{b}=\left(\frac{\delta \mathcal{R}}{\mathcal{R}} \mathbf{1}+\frac{1}{\mathcal{R}^{2}} \sigma\right)^{a}{ }_{b}, \quad h_{2}^{2}=-2 \frac{\delta \mathcal{R}}{\mathcal{R}},
$$

where $\sigma$ is the matrix associated with the vector

$$
\Sigma \equiv \mathbf{X} \wedge \delta \mathbf{X}
$$

and we have used (3.9) and (B.2). Note that (B.6) implies $h=0$.

Let us now compute $\mathcal{F}_{E}^{02}(V)$ for an arbitrary spacetime vector $V^{a}$ (with $\left.V^{2}=0\right)$. It is convenient to first compute the covariant components

$$
\begin{aligned}
\mathcal{F}_{E a 2}(V)= & \frac{1}{2}(\lambda V)_{a}\left(-\partial_{2} h_{2}^{2}+\Gamma_{22}^{2} h_{2}^{2}+\Gamma_{2 c}^{b} h_{b}^{c}\right) \\
& -\frac{1}{2}(\lambda V)_{b} \partial_{2} h_{a}^{b}+\frac{1}{2} h^{2}{ }_{2} \partial_{2}(\lambda V)_{a} \\
= & \frac{1}{2}(\lambda V)_{a}\left[2\left(\frac{\delta \mathcal{R}}{\mathcal{R}}\right)^{\prime}+2 \frac{\mathcal{R}^{\prime} \delta \mathcal{R}}{\mathcal{R}^{2}}+\frac{1}{2} \operatorname{Tr}\left(\lambda^{-1} \lambda^{\prime} \lambda^{-1} \delta \lambda\right)\right] \\
& -\frac{1}{2}(\lambda V)_{b}\left(\lambda^{-1} \delta \lambda\right)^{\prime b}-\frac{\delta \mathcal{R}}{\mathcal{R}}(\lambda V)_{a}^{\prime} .
\end{aligned}
$$


This leads to

$$
\begin{aligned}
\mathcal{F}_{E}^{02}(V)= & \frac{1}{2} \zeta^{2}\left\{V\left(\mathbf{X} \cdot \delta \mathbf{X}^{\prime}+2 \mathcal{R}^{\prime} \delta \mathcal{R}\right)\right. \\
& \left.+V \lambda\left(-\sigma^{\prime}+2 \frac{\mathcal{R}^{\prime}}{\mathcal{R}} \sigma-2 \mathcal{R} \delta \mathcal{R} \lambda^{-1} \lambda^{\prime}\right) \lambda^{-1}-2 \mathcal{R} \delta \mathcal{R} V^{\prime}\right\}^{0}
\end{aligned}
$$

After some algebra with use of (3.9) , (B.2) and (3.8) one finds

$\lambda\left(-\sigma^{\prime}+2 \frac{\mathcal{R}^{\prime}}{\mathcal{R}} \sigma-2 \mathcal{R} \delta \mathcal{R} \lambda^{-1} \lambda^{\prime}\right) \lambda^{-1}=\tau^{0}\left(2 \mathcal{R}^{\prime} \delta \mathcal{R} 1-\delta \ell\right) \tau^{0}=\left(-2 \mathcal{R}^{\prime} \delta \mathcal{R} 1-\delta \ell^{t}\right)$,

and finally

$$
\mathcal{F}_{E}^{02}(V)=\frac{1}{2} \zeta^{2}\left[\left(\mathbf{X} \cdot \delta \mathbf{X}^{\prime}-\delta \ell\right) V-2 \mathcal{R} \delta \mathcal{R} V^{\prime}\right]^{0} .
$$

Now we move on to the computation of the Cotton part of the superpotential. Using $\xi^{\prime a}=0$, the vector defined in (2.18) takes the form 5

$$
\eta^{a}=\frac{\zeta}{2} \epsilon^{a b} \xi_{b}^{\prime}=-\frac{\zeta}{2}\left(x^{\prime} \xi\right)^{a}
$$

Replacing in (B.11), and using again (3.9) one gets

$$
\mathcal{F}_{E}^{02}(\eta)=\frac{1}{4} \zeta^{3}\left\{\left(\delta \mathbf{L} \cdot \mathbf{X}^{\prime} \underline{1}-\left[\delta\left(\mathbf{X}^{\prime} \wedge \mathbf{L}\right)+\frac{1}{2} \delta\left(\mathbf{X}^{\prime 2}\right) \mathbf{X}-\delta\left(\mathbf{X}^{2}\right) \mathbf{X}^{\prime \prime}\right]\right) \xi\right\}^{0} .
$$

The next term in (2.21) contributes

$$
\begin{aligned}
& \frac{1}{\sqrt{|g|}}(\lambda \xi)_{c}\left(\epsilon^{0 b} \delta R_{b}^{c}-\frac{1}{4} \epsilon^{0 c} \delta R\right)=\frac{1}{2} \zeta^{3}\left[\xi \lambda\left(\delta \ell^{\prime}+\frac{1}{4} \delta\left(\mathbf{X}^{\prime 2}\right) \mathbf{1}\right) \tau^{0}\right]^{0} \\
& =\frac{1}{2} \zeta^{3}\left[\left(\left[\mathbf{X} \wedge \delta \mathbf{L}^{\prime}+\frac{1}{4} \delta\left(\mathbf{X}^{\prime 2}\right) \mathbf{X}\right]-\mathbf{X} \cdot \delta \mathbf{L}^{\prime} \underline{1}\right) \xi\right]^{0} \\
& =\frac{1}{2} \zeta^{3}\left[\left(\left[\delta\left(\mathbf{X} \wedge \mathbf{L}^{\prime}\right)-\frac{1}{2} \delta\left(\mathbf{X}^{2}\right) \mathbf{X}^{\prime \prime}+\left(\mathbf{X}^{\prime \prime} \cdot \delta \mathbf{X}\right) \mathbf{X}\right.\right.\right. \\
& \left.\left.\left.+\frac{1}{4} \delta\left(\mathbf{X}^{\prime 2}\right) \mathbf{X}\right]-\mathbf{X} \cdot \delta \mathbf{L}^{\prime} \underline{1}\right) \xi\right]^{0} \text {. }
\end{aligned}
$$

Finally, the last term in (2.21) contributes

$$
\begin{aligned}
\frac{1}{2 \sqrt{|g|}} \epsilon^{0 a}(\lambda \xi)_{a}\left(h^{b}{ }_{c} R_{b}^{c}+h^{2}{ }_{2} R^{2}{ }_{2}\right) & =\frac{1}{2} \zeta^{3}\left(\frac{1}{\mathcal{R}^{2}} \boldsymbol{\Sigma} \cdot \mathbf{L}^{\prime}+\frac{\delta \mathcal{R}}{\mathcal{R}}\left[\mathbf{X}^{\prime 2}-\left(\mathcal{R} \mathcal{R}^{\prime}\right)^{\prime}\right]\right)(x \xi)^{0} \\
& =-\frac{1}{2} \zeta^{3} \mathbf{X}^{\prime \prime} \cdot \delta \mathbf{X}(x \xi)^{0}
\end{aligned}
$$

\footnotetext{
${ }^{5}$ Our convention $\epsilon^{012}=-1$ implies $\epsilon^{a b 2}=-\epsilon^{a b}$ with $\epsilon^{01}=+1$.
} 
where we have used

$$
\boldsymbol{\Sigma} \cdot \mathbf{L}^{\prime}=\left(\mathbf{X} \cdot \mathbf{X}^{\prime \prime}\right) \mathcal{R} \delta \mathcal{R}-\mathcal{R}^{2}\left(\mathbf{X}^{\prime \prime} \cdot \delta \mathbf{X}\right) .
$$

Collecting( (B.13), (B.14) and( $(\overline{B .15})$, we end up with a simple expression for the Cotton superpotential

$$
\mathcal{F}_{C}^{02}(\xi)=\frac{1}{2} \zeta^{3}\left\{\left(\delta\left[\mathbf{X} \wedge \mathbf{L}^{\prime}\right]-\frac{1}{2} \delta\left[\mathbf{X}^{\prime} \wedge \mathbf{L}\right]+\frac{1}{2} \mathbf{X}^{\prime} \cdot \delta \mathbf{L}-\mathbf{X} \cdot \delta \mathbf{L}^{\prime}\right) \xi\right\}^{0} .
$$

\section{References}

[1] S. Deser, R. Jackiw and S. Templeton, Phys. Rev. Lett. 48 (1982) 975; Ann. Phys., NY 140 (1982) 372.

[2] M. Bañados, C. Teitelboim and J. Zanelli, Phys. Rev. Lett. 69 (1992) 1849; M. Bañados, M. Henneaux, C. Teitelboim and J. Zanelli, Phys. Rev. D 48 (1993) 1506.

[3] N. Kaloper, Phys. Rev. D 48 (1993) 2598.

[4] K. Ait Moussa, G. Clément and C. Leygnac, Class. Quantum Grav. 20 (2003) L277.

[5] Y. Nutku, Class. Quantum Grav. 10 (1993) 2657.

[6] M. Gürses, Class. Quantum Grav. 11 (1994) 2585.

[7] R. Arnowitt, S. Deser and C.W. Misner, The dynamics of general relativity, in "Gravitation: An Introduction to Current Research", ed. L. Witten (Wiley, New York, 1962).

[8] J.D. Brown and J.W. York, Phys. Rev. D 47 (1993) 1407.

[9] S. Deser and X. Xiang, Phys. Lett. B 263 (1991) 39.

[10] I.L. Buchbinder, S.L. Lyakhovich and V.A. Krykhtin, Class. Quantum Grav. 10 (1993) 2083.

[11] M.-I. Park, "Holography in three-dimensional Kerr-de Sitter space with a gravitational Chern-Simons term", arXiv:0705.4381 [hep-th].

[12] G. Clément, Phys. Rev. D 68 (2003) 024032.

[13] A.A. García, F.W. Hehl, C. Heinicke and A. Macías, Phys. Rev. D 67 (2003) 124016.

[14] J.H. Cho, J. Korean Phys. Soc. 44 (2004) 1355.

[15] S. Deser, I. Kanik and B. Tekin, Class. Quantum Grav. 22 (2005) 3383. 
[16] S. Ölmez, Ö. Sarioğlu and B. Tekin, Class. Quantum Grav. 22 (2005) 4355 .

[17] P. Kraus and F. Larsen, JHEP 0601 (2006) 022.

[18] S.N. Solodukhin, Phys. Rev. D 74 (2006) 024015.

[19] C. Møller, Ann. Phys. (NY) 4 (1958) 347.

[20] A. Komar, Phys. Rev. 113 (1959) 934.

[21] C.-M. Chen and J.M. Nester, Grav. Cosm. 6 (2000) 257.

[22] L.F. Abbott and S. Deser, Nucl. Phys. B 195 (1982) 76.

[23] S. Deser and B. Tekin, Phys. Rev. Lett. 89 (2002) 101101; Phys. Rev. D 67 (2003) 084009.

[24] S. Deser and B. Tekin, Class. Quantum Grav. 20 (2003) L259.

[25] G. Clément, Phys. Rev. D 49 (1994) 5131.

[26] G. Clément, Class. Quantum Grav. 11 (1994) L115.

[27] K. Ait Moussa, G. Clément, H. Guennoune and C. Leygnac, in preparation.

[28] Y. Tachikawa, Class. Quantum Grav. 24 (2007) 737.

[29] A.N. Petrov, Class. Quantum Grav. 22 (2005) L83.

[30] S. Weinberg, "Gravitation and Cosmology: Principles and Applications of the General Theory of Relativity" (Wiley, New York, 1972). 